\title{
Globe
}

Revue internationale d'études québécoises

\section{Jocelyn Létourneau, Je me souviens? Le passé du Québec dans \\ la conscience de sa jeunesse, Montréal, Fides, 2014}

\section{Louise Bienvenue}

Volume 17, numéro 2, 2014

URI : https://id.erudit.org/iderudit/1036243ar

DOI : https://doi.org/10.7202/1036243ar

Aller au sommaire du numéro

Éditeur(s)

Globe, Revue internationale d'études québécoises

ISSN

1481-5869 (imprimé)

1923-8231 (numérique)

Découvrir la revue

Citer ce compte rendu

Bienvenue, L. (2014). Compte rendu de [Jocelyn Létourneau, Je me souviens ? Le passé du Québec dans la conscience de sa jeunesse, Montréal, Fides, 2014]. Globe, 17(2), 207-211. https://doi.org/10.7202/1036243ar d'utilisation que vous pouvez consulter en ligne.

https://apropos.erudit.org/fr/usagers/politique-dutilisation/ 


\title{
RECENSIONS
}

\author{
Maxime Raymond-Dufour (éd.) \\ Stéfany Boisvert (éd.)
}

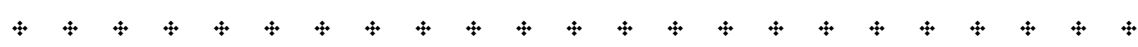

\section{Jocelyn Létourneau}

Je me souviens? Le passé du Québec dans la conscience de sa jeunesse, Montréal, Fides, 2014.

"Si vous aviez à résumer, en une phrase ou une formule, l'aventure historique québécoise, qu'écririez-vous personnellement? " Telle est la question qu'ont posée Jocelyn Létourneau et son équipe à 3475 jeunes issus de toutes les régions du Québec, entre 2003 et 2013. Cette enquête menée dans des classes de $4^{\mathrm{e}}$ et de $5^{\mathrm{e}}$ secondaire, de cégep et d'université a généré un très honorable taux de réponse de 79,2\%. Les données recueillies forment le corpus à partir duquel l'historien explore la conscience historique de la jeunesse québécoise.

Présentées telles qu'elles ont été écrites, les réponses des jeunes, dont voici quelques exemples, ne manquent pas de sel : "On se fait fourrer et il se passe pas grand chose au Québec mais on est bien" (p. 86) ; "Ça juste pris du temps avant que ça l'aboutisse" (p. 68) ; "C'est beau mais c'est trop nationalistis» (p. 69) ; "Un échange de culture qui nous a rendu ouvert d'esprit» (p. 97) ; "Puisque l'histoire est écrite par les vainqueurs, on est chanceux d'en avoir une" (p. 110) ; "Le Québec est très influençable peu importe l'époque" (p. 154); "Un certain nombre de marins partent de France pour aller chercher des épices et arrive $[$ sic $]$ au Québec» (p. 156); "Une histoire qui date depuis 402 ans et qui continue" (p. 209) ; "It's all about the English versus the French» (p. 119).

De toute évidence, l'équipe de Létourneau a dû travailler fort pour classifier ces énoncés et en extraire de l'intelligibilité. Après avoir 
présenté la méthodologie d'enquête (chapitre 1), l'ouvrage expose la morphologie d'ensemble du corpus (chapitre 2), telle qu'elle se dégage d'un classement des énoncés en cinq catégories, chacune exprimant des visions différentes du passé : malheureuses, mixtes, positives, neutres et «autres ». La catégorie dominante est celle du malheur, qui rassemble 35,6\% des résultats. Un pessimisme ambiant s'observerait ainsi dès le $4^{\mathrm{e}}$ secondaire $(2,32 \%)$, avant même que les élèves n'aient suivi le cours d'histoire nationale, et s'accentue tout au cours de la scolarité pour atteindre 48,8\% à l'université.

Les chapitres 3 à 11 décortiquent les réponses selon les catégories de répondants et en fonction de quelques thèmes. On s'intéresse d'abord aux élèves de $4^{\mathrm{e}}$ secondaire (chapitre 3). Moins instruits que leurs aînés, ils ont tendance à privilégier des phrases neutres pour exprimer leur vision mal assurée du passé québécois. L'auteur s'intéresse ensuite aux élèves de $5^{\mathrm{e}}$ secondaire (chapitre 4). Âgés de 16 ou 17 ans et plus autonomes sur le plan intellectuel, ces jeunes formulent des opinions plus affermies. Ils sont désormais près de $40 \%$ à exprimer une vision de l'aventure québécoise classée comme malheureuse. On observe aussi que ces jeunes s'identifient au sujet collectif dont ils parlent, se percevant comme "héritiers et fiduciaires d'une société, d'une nation ou d'un peuple dans la continuité duquel ils veulent se situer» (p. 88). À propos des finissants du secondaire, Létourneau rappelle que seulement $5 \%$ d'entre eux auront l'occasion de suivre des cours d'histoire dans la suite de leur parcours. Pour l'immense majorité, la vision générale du passé québécois acquise au terme du secondaire risque d'être peu ébranlée par la suite.

Puis, suivant les échelons du système éducatif, l'ouvrage se consacre aux étudiants du collégial. Ce segment de l'enquête s'appuie sur un corpus plus restreint, composé de 298 répondants, dont aucun n'est issu de la région montréalaise. À leur sujet, l'auteur constate que près de la moitié (46,3\%) articule une vision à tonalité malheureuse ou victimaire. À l'autre bout du spectre, toutefois, on trouve aussi un nombre plus élevé de cégépiens que d'étudiants du secondaire ayant recours à des phrases positives pour traduire leur appréciation de l'histoire du Québec (p. 93). Enfin, le chapitre 6 met l'accent sur la vision des universitaires. L'historien s'appuie sur l'examen de 509 formules colligées auprès d'universitaires inscrits dans plusieurs disciplines. Sur le plan des thèmes abordés, Létourneau observe que le contenu ne se distingue pas fondamentalement de ce qui était exprimé par les élèves et les étudiants des ordres inférieurs. On a affaire ici, soutient-il, à un même régime énonciatif, et la proportion des phrases victimaires ou malheureuses frôle, dans leur cas, les $50 \%$. 
Considérant l'importance réitérée du conflit anglophones-francophones dans la trame historique décrite par les jeunes, il était incontournable que l'ouvrage réserve une section aux répondants des établissements d'enseignement anglophones (chapitre 7). Ces jeunes, au nombre de 462, sont issus du secondaire et de l'université (aucun ne provient du cégep). Ils expriment une vision très négative du passé québécois. Chez les universitaires (tous des étudiants en histoire), ils sont $56 \%$ à inscrire leur propos au registre du récit malheureux (p. 117). Très peu, note l'auteur, s'identifient au "nous " québécois.

La suite de l'étude discute de variables dont les effets se sont révélés plus marginaux : le lieu de résidence (chapitre 8) et le sexe des répondants (chapitre 9). De toute évidence, souligne Létourneau, la communauté d'histoire et de mémoire (franco-québécoise ou anglo-québécoise) est le facteur principal qui détermine l'orientation des récits (p. 144). Le chapitre 10, pour sa part, souligne la quasi-absence des Autochtones et du Canada dans la variété des thèmes abordés par les jeunes.

Dans l'avant-dernier chapitre, l'auteur tente une comparaison entre la vision du passé exprimée par les jeunes et celle de la population en général. L'exercice est forcément imparfait puisqu'il est établi à partir d'emprunts partiels à d'autres études. Il en ressort néanmoins une idée forte : les thèmes tragiques du "manque » et de "l'inachevé» caractérisent aussi l'expression des adultes. De cette forte corrélation, Létourneau conclut à l'existence d'une matrice mémorielle puissante qui conditionne durablement le rapport des Québécois à leur passé.

Quiconque s'intéresse à ces questions sait qu'à l'automne 2007, un nouveau programme d'histoire et d'éducation à la citoyenneté a été mis en place en $3^{\mathrm{e}}$ et en $4^{\mathrm{e}}$ secondaires. Pour tenter d'en mesurer l'impact, l'équipe de Létourneau a sondé un millier d'élèves et d'étudiants " réformés ", entre le printemps et l'automne 2012 et l'hiver 2013. Si quelques changements ressortent, ils se révèlent plutôt rares : "sauf en $4^{\mathrm{e}}$ secondaire, il est toujours une plus grande proportion de jeunes qui dépeignent le passé du Québec par le biais d'une représentation à connotation malheureuse" (p. 201). S'appuyant sur ce constat, l'auteur ne manque pas de répondre aux détracteurs du cours réformé: qu'ils apaisent leurs craintes, car le programme est loin d'avoir induit automatiquement « une vision allègre, joviale ou rosie du passé québécois» (p. 203).

D’une ampleur inédite, l'enquête réalisée par Létourneau et son équipe est assurément une contribution de taille à la compréhension de la conscience historique des Québécois. Elle fournit une riche base empirique 
pour étayer les discussions - nombreuses et enflammées - sur le sujet. Rédigé de manière limpide (ce qui est un tour de force s'agissant d'un commentaire de statistiques), l'ouvrage ne camoufle pas les inévitables faiblesses du corpus recueilli, qui souffre parfois d'asymétrie, et les limites de la méthodologie. À cet égard, d'ailleurs, il nous faut exprimer un regret. Toute l'étude repose sur des réponses à la question finale d'un plus large questionnaire soumis aux jeunes. Ainsi, avant de produire leur phrase synthèse, les sondés étaient invités à établir de courts récits (de trois paragraphes à trois pages) de l'histoire du Québec. On ne sait pourquoi l'auteur a écarté ce matériau qui semble pourtant potentiellement plus substantiel que les courtes phrases retenues pour l'analyse. Les locutions sur lesquelles repose l'analyse, en effet, sont souvent très courtes et peu évocatrices - «Sa pas été une histoire facile " (p. 96), «De l'ostinage par-dessus de l'ostinage» (p. 209). Il semble même que plusieurs jeunes (près de $15 \%$ dans certaines catégories) se soient contentés d'inscrire la devise du Québec ou quelque chose d'approchant en guise de réponse. À plusieurs reprises, l'historien nous a semblé d'ailleurs fort généreux et indulgent de prêter autant de hauteur à des phrases qui témoignaient difficilement d'un véritable regard réflexif sur le passé.

Il y a longtemps que Jocelyn Létourneau se désole de la vision victimaire que les Québécois reconduisent à l'égard de leur passé. Or la lecture de cet ouvrage éveille une question : la conscience malheureuse du passé est-elle une névrose spécifiquement québécoise? L'étude devrait mieux distinguer ce qui relève de l'appréciation généralement sombre du passé d'une mélancolie plus directement associée à l'inaccomplissement national. Si celle-ci, à n'en pas douter, appartient à la catégorie du malheur, elle ne saurait en résumer l'ensemble. Autrement dit, alors que certaines locutions traduisent une tristesse manifeste à l'égard d'un Québec jamais parvenu à son indépendance, d'autres expressions douloureuses renvoient davantage, selon moi, à ce lieu commun selon lequel nous sommes les héritiers d'un passé difficile. Ainsi, une phrase comme «Beaucoup de misères pour une Belle province " n'entre pas nécessairement dans la catégorie des commentaires désolés sur la question nationale. Évoquer son histoire, personnelle ou collective, comme un chemin parsemé d'épreuves et de contrariétés est un patron narratif généralisé. La lire comme un constant travail de préservation de Soi par rapport à l'Autre l'est tout autant. Nombre de peuples se décrivent assurément dans ces termes, même si quelques nations privilégiées se gargarisent plus impérialement de récits glorieux. S'agissant de l'aventure humaine, une telle lecture n'est d'ailleurs pas réservée au passé. De l'actualité, on dira aussi que "ça va mal», que l'adversité est constante... C'est par rapport à de 
tels enjeux que l'auteur, me semble-t-il, pèche en ne mobilisant pas suffisamment, à des fins comparatives, les travaux sur la mémoire réalisés dans d'autres cadres nationaux, qui, souligne-t-il, sont pourtant nombreux (p. 21). Ainsi vaudrait-il mieux mener plus loin l'exercice comparatif avant de conclure à un mal proprement québécois, alimenté par des rhétoriciens habiles.

Soulevant des questions nombreuses sur le rapport au passé des Québécois et sur le rôle complexe de l'école dans la construction de celui-ci, l'ouvrage de Jocelyn Létourneau stimule assurément notre réflexion sur ces enjeux importants.

Louise Bienvenue

Université de Sherbrooke

\section{Denis Goulet et Robert Gagnon \\ Histoire de la médecine au Québec, 1800-2000. De l'art de soigner à la science de guérir, Québec, Septentrion, 2014.}

Ce n'est pas trop tôt! On attend depuis longtemps un ouvrage de synthèse sur l'histoire des médecins et de la médecine au Québec. Les synthèses précédentes de Jacques Bernier ou de Rénald Lessard étaient de grande qualité mais ne couvraient pas le $\mathrm{XX}^{\mathrm{e}}$ siècle, période-clef de l'évolution de la médecine s'il en est. Bref, le livre de Goulet et Gagnon comble un réel besoin.

L'ouvrage se divise en deux parties: l'une sur le XIX ${ }^{\mathrm{e}}$ siècle, l'autre sur le $\mathrm{XX}^{\mathrm{e}}$. Difficile de faire plus simple. La première partie s'étale sur cinq chapitres et 135 pages. On y aborde les thèmes classiques de l'histoire médicale : la diversité du marché des soins au XIX ${ }^{e}$ siècle (le médecin n'est qu'une variété de soignants parmi d'autres), la diffusion des idées anatomocliniques et bactériologiques, la standardisation et la modernisation des études médicales, le ralliement de la profession autour du Collège des médecins, qui conquiert, à la fin du siècle, des monopoles légaux décisifs. Sont aussi abordés l'essor relatif de la santé publique, l'état des institutions de soins au XIX siècle et la médicalisation de la folie.

La portion portant sur le $\mathrm{XX}^{\mathrm{e}}$ siècle est plus longue : onze chapitres et 280 pages. On commence par dresser un portrait de la situation au début du siècle, en s'attardant sur l'entrée des médecins dans les hôpitaux et sur les 